\title{
Médiévales
}

Langues, Textes, Histoire

57 | automne 2009

Langages politiques, $\mathrm{XII}{ }^{\mathrm{e}}-\mathrm{XV}^{\mathrm{e}}$ siècle

\section{Le nom Machiavel}

\section{Étienne Anheim}

\section{(2) OpenEdition}

\section{Journals}

Édition électronique

URL : https://journals.openedition.org/medievales/5830

DOI : $10.4000 /$ medievales. 5830

ISSN : $1777-5892$

\section{Éditeur}

Presses universitaires de Vincennes

\section{Édition imprimée}

Date de publication : 20 décembre 2009

Pagination : 151-159

ISBN : 978-2-84292-241-2

ISSN : 0751-2708

\section{Référence électronique}

Étienne Anheim, «Le nom Machiavel », Médiévales [En ligne], 57 | automne 2009, mis en ligne le 18 janvier 2012, consulté le 22 avril 2022. URL : http://journals.openedition.org/medievales/5830 ; DOI : https://doi.org/10.4000/medievales.5830 
Médiévales 57, automne 2009, p. 151-160

\section{Étienne ANHEIM}

\section{LE NOM MACHIAVEL}

Le hasard, qui gouverne un peu plus de la moitié de nos actions, a mis sur les étals des librairies de l'automne 2008 deux livres voisins, voire cousins, Léonard et Machiavel de l'historien Patrick Boucheron et Le rêve de Machiavel du romancier Christophe Bataille ${ }^{1}$. En découvrant ces deux livres, la première impression est d'avoir affaire à deux projets opposés, des procédés littéraires au service d'un discours historique chez Boucheron là où, chez Bataille, ce serait le contraire. Mais rapidement, le lecteur ne peut qu'être sensible à ce qui rassemble les deux ouvrages, le souci de décrire le retournement des temps et de saisir le sombre éclat de la Renaissance italienne - de saisir surtout, confronté à Léonard ou à la peste, la figure de Machiavel.

Le personnage s'impose et contraint le récit: pour l'historien, rien que de très normal; c'est plus singulier pour le romancier qui accepte de tenir en bride son imagination et désigne parfois, au passage, une bifurcation possible. Lorsque le prêtre monologue devant Machiavel sans dire d'abord s'il le reconnaît, il s'interroge : " peut-être ai-je croisé l'homme qui se cache dans la ville ? L'homme dont on parlait tant ? Peut-être ai-je croisé la cause de tout, comme ils disent, après qu'ils ont brûlé les filles de rue, les juifs, les enfants, leur bourreau ? Oui... Peut-être ai-je croisé Machiavel qu'on doit tuer pour éteindre la peste ${ }^{2}$ ?» Peut-être croise-t-on ici le désir de l'écrivain d'un autre livre, d'une chasse à Machiavel dont le destin serait lié à la peste par un sortilège inconnu et qu'on devrait sacrifier tel un bouc-émissaire ? Ce

1. Certains éléments de ce texte ont été présentés et discutés au séminaire d'histoire médiévale de l'Université de Versailles/Saint-Quentin-en-Yvelines le 10 novembre 2008 en présence de Patrick Boucheron, et au débat coordonné par Marie Lezowski et Antoine Lilti à l'ENS de la rue d'Ulm le 27 janvier 2009 dans le cadre de la semaine « Histoire et fiction », organisée par Nadeije Laneyrie Dagen, Gilles Pécout et Antoine Lilti. Je remercie beaucoup les deux auteurs, les collègues et les étudiants qui ont participé à ces discussions, ainsi que Sophie de Closets et Valérie Theis.

2. C. Bataille, Le rêve de Machiavel, Paris, Grasset, 2008, p. 130. 
n'est pourtant pas le livre dans lequel Bataille s'engage - sans doute ne pouvait-il naître que d'un héros anonyme traversant la Toscane comme le hussard de Giono la Provence de 1832.

Machiavel est une ancre qui empêche la dérive du discours, ou plutôt son nom, car il y a un «nom Machiavel » comme il y a une « œuvre Machiavel » ${ }^{3}$. Autour de cette poétique des noms de l'histoire explorée par Jacques Rancière se noue le lien qui unit le romancier et l'historien, d'une manière presque trop évidente tant il occupe le devant de la scène ${ }^{4}$ :

Les historiens qui ont voulu rompre avec la vieille chronique pour donner, autant que possible, à l'histoire la rigueur d'une science ont dû se battre avec les présupposés et les équivoques attachés au nom même d'histoire. Une histoire, au sens ordinaire, c'est une série d'événements qui arrivent à des sujets généralement désignés par des noms propres. Or la révolution de la science historique a justement voulu révoquer le primat des événements et des noms propres au profit des longues durées et de la vie des anonymes. [...] Ce qu'on se dispense de considérer est simplement ceci : l'histoire n'est, en dernière instance, susceptible que d'une seule architecture: il est arrivé une série d'événements à tel ou tel sujet. On peut choisir d'autres sujets : la royauté au lieu des rois, les classes sociales, la Méditerranée ou l'Atlantique plutôt que les généraux ou les capitaines. On n'en affrontera pas moins le saut dans le vide contre lequel les rigueurs d'aucune discipline auxiliaire n'apportent de garantie : il faut nommer des sujets, il faut leur attribuer des états, des affections, des événements.

Le sujet, ce nom Machiavel, présent dans les deux titres, est ici omniprésent - ce qui n'est que le reflet de ce qu'il est devenu pour nous. Comme l'écrit Patrick Boucheron, qui l'appelle Niccolo Machiavelli à la première page de son livre avant de ne plus jamais le nommer que Machiavel, «pour l'heure, son visage n'est pas encore recouvert de ce masque repoussant qui porte, en Europe, le nom de Machiavel et qui, plus effrayant encore que celui des débauches et des tares borgiaques, est pour la postérité comme la gueule du monstre en politique ${ }^{5}$. Christophe Bataille, en écho, en joue ostensiblement : «qui sait ce qu'il y a dans un nom ? ${ }^{6}$ demande un personnage à son héros, qui lui-même dit à une femme qu'il veut séduire et qui l'a reconnu : «ne t'inquiète pas pour Machiavel. J'ai usurpé son nom. C'est un vêtement. Un vêtement de prince pour la peste. Disons que tu es la reine de cette ville et que suis Machiavel. C'est comme un jeu $\gg^{7}$.

C'est ce nom qui a attiré et rapproché les deux auteurs, poursuivant chacun à sa façon le même projet, retrouver l'homme derrière le nom, parler

3. C. Lefort, Le travail de l'œuvre Machiavel, Paris, Gallimard, 1972.

4. J. RANCIÈRE, Les noms de l'histoire. Essai de poétique du savoir, Paris, Seuil, 1992, p. 7-9.

5. P. Boucheron, Léonard et Machiavel, Lagrasse, Verdier, 2008, p. 9-10.

6. C. Bataille, Le rêve de Machiavel, p. 38.

7. Ibid., p. 58. 
d'un Machiavel qui ne serait pas encore le «nom Machiavel»: «je prends Machiavel à ses mots. Je le prends au temps et à sa légende. J'en fais un homme $»^{8}$. Autour de lui, les deux livres proposent une succession de noms propres devenus presque communs et que les auteurs mettent en branle pour tenter de les faire échapper au déterminisme du temps passé, au fait que nous connaissons déjà la fin de l'histoire : César Borgia ou Léonard chez Boucheron, Laurent de Médicis et tant d'autres chez Bataille, jusqu' au trompe-l'œil de Jacobus Fontialis ${ }^{9}$. Se dévoile le dessein commun de travailler à rebroussepoil du temps passé, ce qui n'est pas sans paradoxe puisque ce sont les historiens et les écrivains qui ont fait de l'homme Machiavel un nom.

Ainsi Boucheron et Bataille n'écrivent pas tant sur l'auteur du Prince qu'ils ne réécrivent à l'envers de ceux qui ont noirci des pages sur lui et sur les premières décennies du $\mathrm{XVI}^{\mathrm{e}}$ siècle italien. Le lecteur éprouve une sensation de déjà-vu, une sorte de vertige du palimpseste - qui n'est peut-être du reste qu'un fruit de son imagination et de sa propre culture littéraire : on passerait de «la vie en mouvement» de l'CEuvre au noir de Marguerite Yourcenar à l'Italie des Mémoires de Blaise de Monluc ; de Giono, qui justement préfaça Monluc, à Michelet qui est la source du Rêve de Machiavel. Singulièrement, ces réécritures sont à front renversé ; on entend le romancier Giono se contraignant à écrire Le Désastre de Pavie chez Boucheron, là où l'empreinte de La Sorcière et d'un historien devenant écrivain semble si forte chez Bataille.

Cet écart rappelle que malgré toutes les consonances, ces deux livres ne se confondent pas et n'appartiennent peut-être qu'en apparence au même univers. Pourquoi les écrire ? Pour dire «la qualité » de ces temps de tumulte et l'effacement d'un homme derrière son nom, sans doute, et pour le plaisir d'écrire, aussi. Mais pas seulement : si Machiavel a appris à des générations à se servir des hommes, l'historien et le romancier se servent ici de lui tout en le rendant à son humanité. Chez Patrick Boucheron, Machiavel sert de révélateur, au sens presque photographique, à Léonard qui est le grand personnage du livre: d'une certaine manière, l'historien ajoute un nouveau chapitre à une œuvre qu'il écrit depuis au moins son l'article sur la statue équestre de Francesco Sforza et qui se poursuit dans son livre sur Milan ${ }^{10}$, une œuvre dont Léonard serait le héros sans jamais qu'il s'agisse d'une biographie et qui chercherait à distiller «l'esprit-du-temps » comme on distille l'esprit-de-vin, au centre d'un triangle composé par l'art, la science et le pouvoir du prince - et on note, comme une préfiguration, que Machiavel et Léonard s'étaient déjà croisés chez Boucheron il y a quelques années, sous

8. Ibid., p. 141

9. Ibid., p. 201.

10. P. Boucheron, «La statue équestre de Francesco Sforza: enquête sur un mémorial politique », Journal des Savants, juillet-décembre 1997, p. 421-499; Le pouvoir de bâtir. Urbanisme et politique édilitaire à Milan (XIV $-X V^{e}$ s.), Rome, Coll. de l'EFR, 239, 1999. 
l'égide de Foucault ${ }^{11}$. Chez Christophe Bataille, Machiavel est aussi le masque sous lequel se prolonge, après celui de Jocelyn Simarre, un même projet d'écriture. Livre après livre, il s'agit de détruire l'élégance originelle d'Annam, d'Absinthe et du Maître des heures au profit d'une langue vive et dure qui exprime la destruction et la répugnance du monde et des corps ${ }^{12}$, qui rythme une danse macabre de squelettes surgis du sillon creusé par un véritable auteur - si ce mot qualifie celui qui poursuit, même si cela doit surprendre son lecteur, son idée de ce qu'est l'écriture.

C'est peut-être alors que se fait jour la plus grande différence : Machiavel est un prétexte, pour l'historien, à saisir le temps qui passe, là où pour l'écrivain, il est un miroir pour se saisir lui-même, dans ses fantasmes et ses obsessions. Les deux ouvrages s'inscrivent dans deux mondes parallèles qui se ressemblent tout en différant fondamentalement. Cela rassurera les uns, attristera les autres : il n'y a aucun effet de brouillage tel qu'on en rencontre chez le Giono de Pavie ou la Yourcenar des Mémoires d'Hadrien, justement : le livre de l'historien reste toujours un livre d'histoire et le roman ne devient jamais autre chose qu'un roman - et surtout pas un roman historique.

Le prétexte de Bataille est le dernier amour de Machiavel tel qu'il est rêvé par Michelet dans le tome VIII de son Histoire de France (Réforme, 1508-1547) ${ }^{13}$, mais en réalité, cet argument ne tient qu'une place limitée dans le livre. Point de départ de l'auteur peut-être, l'épisode est relégué dans le dernier tiers de l'ouvrage, tandis que dans les deux premiers, Machiavel vagabonde en compagnie de la peste et de la mort dans la Toscane de 1527.

11. P. BOUChERON, «Techniques hydrauliques et technologies politiques : histoires brèves d'ingénieurs au service du duc de Milan à la fin du XV siècle », Mélanges de l'École française de Rome - Moyen Âge, 2004/2, t. 116, p. 803-819.

12. C. Bataille, Annam, Paris, Arléa, 1993 ; Absinthe, Paris, Arléa, 1994 ; Le maître des heures, Paris, Grasset, 1997.

13. Jules Michelet, Histoire de France, t. VIII : Réforme (1508-1547), Paris, Flammarion, 1895 , p. 192 : «Sur les tombes qui entourent l'église, il trouve une jeune femme échevelée qui se frappe le sein. Il avance, non sans quelque crainte; il console, interroge. Elle répond, s'épanche, elle conte en paroles hardies (les morts n'ont peur de rien), en lamentations effrénées, les joies conjugales qu'elle n'aura plus. Ce disant, elle pâme. Est-elle morte ? Pestiférée ou non, Machiavel la délace et desserre, "quoiqu'elle ne fût pas très serrée". Elle revient alors, et jure qu'elle n'a plus souci d'elle, de moeurs ni de pudeur. Là-dessus, un sermon équivoque du bon apôtre, qui prêche la décence des plaisirs secrets. C'est l'horreur sur l'horreur ! la mort entremetteuse !... Ailleurs, à Santa-Maria-Novella, sur les degrés de marbre de la grande chapelle, il trouve, sous de longs vêtements, une admirable veuve. Suit la description, laborieuse, mythologique, de cette divinité. Morceau sensuel, triste, qui sent le vieillard et l'effort. Cupidon, Vénus, les Hespérides, ne réchauffent pas tout cela. Moins froid le marbre funéraire où siège cette idole de mort. Machiavel près d'elle essaye son éloquence. Il n'en faut pas beaucoup. Elle est tout d'abord consolée. La différence d'âge qu'il avoue ne l'arrête guère. La fortune qu'il prétend avoir, les soins et l'amitié, c'est tout ce qu'il faut à la belle. Elle se laisse tout doucement ramener. Un moine accourt. Mais le traité est fait : "Mon coeur, dit Machiavel, est maintenant chez elle, et mon âme est restée dans ses noirs vêtements". Sa vie y reste aussi, un mois ou deux après il meurt. » 
Plutôt qu'un dernier amour, c'est une vanité que cherche à peindre Christophe Bataille, une nature morte ou presque, en train de mourir plus exactement, ce qui devient le motif de toute son écriture. Les premières pages du livre, en proposant une méditation qui s'affranchit par un recours fréquent au style indirect de toute situation du discours, mêlent inextricablement la voix de l'auteur et celle de Machiavel. Le romanesque est réduit à la portion congrue : il fuit Florence, bat la campagne, arrive dans une autre ville dont on ne saura jamais le nom. Tout le livre s'écrit dans cette fuite, et Machiavel lui-même revient sans cesse sur ses pas par la pensée, se rappelant Florence, remontant vers son enfance. À chaque moment sous la plume de Bataille, le temps revient sur lui-même, ce qui accélère encore sa course : c'est l'écriture ellemême qui devient réflexion sur le retournement du monde au tournant de la Renaissance.

Mais Machiavel a-t-il bien quitté Florence ? Comme chez Calderón, comme le laisse entendre le titre, tout cela n'est peut-être qu'un rêve. Rien ne dit que le livre entier n'est pas un songe fiévreux, celui d'un homme en train de mourir de la peste à Florence et qui rêve de salut. La prose heurtée de Bataille montrerait alors que le besoin de sauver une jeune fille, cet investissement d'une pureté idéale, ne pourrait être qu'une illusion, renvoyant encore une fois à l'impureté essentielle du monde réel. Avec Machiavel et les nouveaux temps qui s'ouvrent devant lui, la mort est sans horizon; l'écriture est sans merveilleux, elle a épuisé son éclat et tous ses effets sont destinés à l'échec. Renoncer aux enchantements de l'écriture ainsi que le fait Bataille, c'est se donner les moyens de parler de l'aube d'un monde désenchanté. Violetta, cette fameuse jeune fille qui domine la fin du livre, n'en finit pas de mourir, comme la langue de l'auteur : elles sont condamnées à un pourrissement sans fin qui sont la matière même de l'œuvre. L'écriture est tendue vers sa propre mort par putréfaction, mais ne s'arrête jamais, telle la flèche de Zénon d'Élée qui se rapproche toujours plus de son but sans jamais l'atteindre: voilà qui éclaire aussi le pourquoi de Machiavel, qui incarne cette flèche, ce temps orienté, mais vers rien si ce n'est sa disparition - une

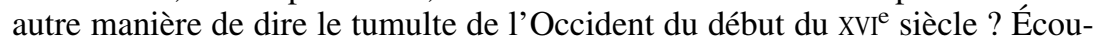
tons encore Bataille: «Les uns diront: c'est le travail de l'historien de connaître Machiavel. À quoi sert de rêver? Les autres diront : que faire de cet homme mort il y a tant d'années ? N'y a-t-il pas des morts récents ? Des morts bien à nous ? N'y a-t-il pas des découvertes, des prophéties, des inventions, des images, et même des pensées qui sont de notre temps? Aujourd'hui mange hier ${ }^{14}$.

Dans Léonard et Machiavel, l'action est tout autre mais le théâtre est le même, car là aussi, « aujourd'hui mange hier ». En neuf chapitres, Patrick Boucheron raconte un chassé-croisé sans début ni fin, concentré entre 1502 
et 1505 , cherchant à montrer comment, de l'entourage de César Borgia à la Florence des Médicis, les parcours de Léonard de Vinci et de Machiavel se sont entrecroisés autour de préoccupations communes et de lieux partagés, sans qu'on puisse jamais être sûr de ce que furent leurs rapports. La réception critique, très favorable, a mis d'abord l'accent sur la dimension imaginaire de l'œuvre, ce qui est l'effet d'un premier chapitre qui joue avec le lecteur en présentant la rencontre possible de Léonard et Machiavel à Urbino après la prise de la ville par César Borgia, en 1502. Pierre Assouline souligne ainsi «le croisement entre essai et récit historique » et songe à Michelet disant qu' « il faut faire parler les silences de l'histoire », ce qui nécessite « un historien au tempérament de dramaturge et à la plume d'écrivain $»^{15}$. Claire Judde de la Rivière, dans Le Monde des Livres, voit Patrick Boucheron «braver les silences, les ombres et les absences, et explorer ce qui a disparu ou qui n'a jamais été », montrant à quel point « vérité et fiction se côtoient, s'observent », révélant le trouble de l'historienne face à ce texte insaisissable, pour finir par prendre une position plus tranchée en citant Salman Rushdie, "quelques libertés ont été prises avec les faits historiques, dans l'intérêt de la vérité » ${ }^{16}$. En complément de ces lectures qui mettent soulignent la dimension littéraire du livre, on peut aussi essayer de le lire comme un livre d'histoire.

Il prendrait ainsi sa place dans un projet historiographique, contournant le problème de la biographie en ayant recours au procédé des vies croisées, qui sont d'ailleurs, pour mieux se prémunir de l'illusion biographique, sans début ni fin. Léonard et Machiavel, qui sont pour notre regard rétrospectif des précurseurs, redeviennent des contemporains l'un de l'autre. Là où le regard de l'historien a tendance à les déplacer vers l'aval, Patrick Boucheron insiste au contraire pour en faire des êtres de chair et de sang qui agissent dans et sur leur temps, qui est peut-être celui de la «modernité » mais surtout celui de la violence et du fracas: le monde change dans la confrontation entre les États modernes d'Europe du nord et la nouvelle culture développée en Italie, conduisant à une réorganisation symbolique et matérielle des formes de la domination. C'est ici que se fait jour le vrai souci de l'historien : tenter de saisir la gestation d'un mouvement par le ralenti, sur le vif, en oubliant pour un instant le dénouement - d'où la figure de l'inachèvement traversant un livre qui n'a d'ailleurs concrètement pas de fin. Pour Boucheron, la Renaissance est d'abord ce moment de bouleversement qui offre de réordonner le temps et dont nous oublions facilement la part de tumulte, restituée ici à travers des figures anachroniques, au sens où elles sont sorties de leur époque pour continuer à habiter la nôtre.

15. P. Assouline, La république des livres, http://passouline.blog.lemonde.fr/2008/09/01/ une-conversation-dans-la-nuit-durbino/

16. C. JudDe De Larivière, "Quand deux génies se rencontrent chez "Le Prince" », Le Monde des Livres, 5 septembre 2008. 
Cependant, cette position historiographique est loin d'épuiser l'intérêt historique de l'ouvrage et ce par quoi il se distingue fondamentalement du roman : il s'agit aussi et surtout, paradoxalement, d'un livre d'épistémologie, qui s'interroge sur le statut de la connaissance et de l'écriture historique, sans pour autant le dire explicitement. Une lecture attentive montre qu'il n'est jamais question d'invention chez Patrick Boucheron, au contraire : la critique historiographique et documentaire est minutieuse et l'auteur évacue tout romanesque et toute dimension psychologisante. L'écriture est elle-même très précise dans son rapport aux documents, comme le montre l'analyse matérielle du carnet, l'étude iconographique des dessins ou l'attention lexicographique portée aux lettres de Machiavel. Les documents sont intégrés dans l'écriture même, insérés, cités, parfois réécrits au point que l'historien tend à se fondre avec sa documentation : si la lecture dès lors s'apparente parfois à un jeu de cache-cache, on s'aperçoit très vite qu'il n'a rien à voir avec le roman historique et qu'il respecte les règles les plus traditionnelles de la critique.

Mais alors, d'où vient l'étrangeté, et quelle est sa portée ? Pourquoi cet air de famille si prononcé entre Bataille et Boucheron ? C'est qu'il n'y a pas de notes, que l'écriture se dispense de faire appel aux concepts analytiques classiques de l'historien et que le livre n'est pas publié dans une collection d'histoire. Cette série d'écarts par rapport à une norme implicite conduit à s'interroger sur ce qu'est, pour le sens commun, un livre d'histoire : elle met en évidence, dans une sorte d'épistémologie pratique, le point auquel notre jugement est conditionné par des critères formels, ce qui nous ramène à des thèses classiques de la théorie des médiations et de la réception, de l'idée de Donald MacKenzie que la forme a des effets sur le sens ${ }^{17}$ à celle de Marshall MacLuhan selon laquelle «the medium is the message ${ }^{18}$. Le choix de la collection, de l'absence des notes, d'une écriture recherchée produisent une perturbation des critères traditionnels d'identification et donnent lieu à une sorte d'expérience : si ce livre est bien un essai, comme le dit avec raison Pierre Assouline, ce n'est pas au sens générique ; c'est une tentative de jouer avec les frontières.

Mais si un livre d'histoire se définit par un projet intellectuel de démonstration qui ordonne une documentation choisie, Léonard et Machiavel en est assurément un, et la réflexion sur l'écriture qu'il propose est d'abord une réflexion sur le statut de la documentation pour l'historien. Tout commence par un jeu avec le lecteur puisque l'absence de sources est mise en évidence dès le début du livre et que l'historien semble prêt de basculer : «Faut-il se lancer à l'eau, dans le grand bain rafraîchissant de la fiction ? Doit-on laisser la parole au romancier ou au dramaturge qui saura reconstituer, en dialogues

17. D. MacKenzIE, La bibliographie et la sociologie des textes (1986), Paris, Cercle de la Librairie, 1991.

18. M. MacLuhan, Pour comprendre les médias. Les prolongements technologiques de l'homme (1964), Paris, Le Seuil, 1968. 
vifs et imagés, le vraisemblable des paroles échangées ? Patience, car de ce théâtre, nous n'avons pas encore entendu le protagoniste le plus éloquent: son décor ${ }^{19}$. »

Joueur jusqu'au bout, l'auteur ne répond pas à ces questions mais s'arrête, renvoyant à plus tard ce que le lecteur imagine, c'est-à-dire l'entrée dans l'invention. Un peu plus loin, le désir se fait jour à nouveau : "Reste une énigme : pourquoi ne nous disent-ils rien l'un de l'autre ? Cette ignorance mutuelle, que l'on peine à trouver accidentelle, est peut-être la part d'ombre la plus attirante de cette histoire, le vide le plus vertigineux qui donne envie d'y précipiter du discours, non pas de manière trop brusque, non, plutôt en y jetant nos mots, un à un, parcimonieusement, à la manière des enfants au bord des cratères lorsqu'ils visitent les volcans anciens, et qui demeurent à la fois paralysés et vibrants du désir d'y vider leurs poches, pour entendre le bruit que fait la pièce de monnaie lorsqu'elle tombe au fond - et sachant bien d'ailleurs qu'ils n'entendront rien car la fosse est trop vaste. Nommer ce silence est impossible ${ }^{20}$. $\gg$ Encore une fois c'est un fauxdépart et le lecteur, page après page, comprend que jamais l'historien ne hissera la voile du romancier. Ce n'était qu'un jeu, pourtant pas gratuit pour autant.

Patrick Boucheron résiste à la tentation de combler le vide et c'est au contraire autour de lui que se développe le discours de l'historien. Deux images expriment avec force la nature du travail historique. La première est celle du gué : «De cette histoire, on ne saisit que quelques bribes. Des mots rares et secs jetés dans les archives, comme des cailloux dans un ruisseau, qu'il nous faut pourtant traverser à gué, en sautillant de l'un à l'autre ${ }^{21}$. » Lui fait écho la figure du puzzle, qui met en évidence l'hétérogénéité fondamentale des matériaux utilisés pour écrire l'histoire et que le liant des mots a tendance à fondre dans un récit qui se voudrait lisse et continu : «ce n'est pas un puzzle, car les éclats de l'histoire n'attendent pas d'être rapprochés par cet enfant sage et un peu triste, patient et esseulé qui survit dans l'esprit de tout historien; ce n'est pas un puzzle puisque rien ne prouve que ce qui gît brisé fut un jour entier, que ces mots isolés proviennent d'une seule et même phrase, ces éclats arrachés de la roche mère d'une seule intrigue. $\mathrm{Ce}$ n'est pas un puzzle, mais peut-être un gué à traverser pour suivre le cours d'une inquiétude commune ${ }^{22}$. $\gg$ On arrive ainsi au terme de l'expérience historiographique de l'auteur, qui fait du jeu sur la forme du livre l'occasion d'une réflexion sur la nature des documents sollicités par l'historien, en rappelant leur caractère ouvert, leur dispersion et la nécessité de construire un discours qui aille d'un morceau à l'autre de ces tessons de passé toujours

19. P. Boucheron, Léonard et Machiavel, p. 11.

20. Ibid., p. 23.

21. Ibid., p. 79.

22. Ibid., p. 82. 
présents dans notre propre présent sans dissimuler le caractère discontinu et fragmenté du réel ${ }^{23}$.

Ainsi, plutôt qu'une théorie de la pratique, on peut trouver dans ce livre une pratique de la théorie visant à montrer par l'exemple que les principaux compagnons de l'historien sont le vide et le silence. Sans doute seraitil possible de retraduire entièrement le livre dans le langage de l'historiographie et de l'épistémologie. On n'est cependant pas obligé de bouder son plaisir de lecture, qui correspond à un évident plaisir d'écriture de la part de Patrick Boucheron. Certains pourront juger l'écriture trop travaillée ou y voir un obstacle dressé par la personnalité de l'historien entre le passé et le lecteur. On pourra aussi trouver qu'il y a une certaine satisfaction à partager le plaisir certain d'un collègue qui aime écrire. L'historien, quand il se met au travail, doit-il toujours refuser la jouissance ? Voilà aussi la question à laquelle mène la lecture croisée de Bataille et de Boucheron. Les deux livres ne se rencontrent jamais tout à fait, sinon dans le désir d'écriture. Mais il y a, dans ce rapprochement dû aux hasards de l'édition et de notre temps - et qui n'est donc pas tout à fait un hasard - la mise en lumière du fait que ce qui sépare l'historien du romancier n'est pas la fiction, si on évite de réduire ce terme à son sens trivial d'invention. La fiction, la façon, du latin fingo, c'est aussi la fabrique, en l'occurrence du discours; elle est toujours présente, puisqu'il faut bien, à partir de son imagination ou de documents vieux de cinq cents ans, mettre en æeuvre; mais l'écriture, de l'historien au romancier, est soumise à différents ordres de contrainte, à la manière de ces géométries qui peuvent être euclidienne ou bien admettre d'autres axiomes, donnant naissance à des espaces aux caractéristiques à la fois si proches et définitivement dissemblables.

Étienne ANHeim, Université de Versailles/Saint-Quentin-en-Yvelines, Laboratoire ESR Moyen Âge-Temps modernes, 47 bd Vauban, F-78047 Guyancourt

23. É. ANHEIM, «Ouvrir les sources au questionnement», Uso e abuso delle fonti, éd. É. Castelli Gattinara et É. Anheim, Dimensioni e problemi della ricerca storica, 2007/2, p. $20-28$. 
